EPJ Web of Conferences 116, 08003 (2016)

DOI: $10.1051 /$ epjconf/201611608003

(C) Owned by the authors, published by EDP Sciences, 2016

\title{
Neutrino-nucleon cross sections at energies of Megaton-scale detectors
}

\author{
A. Gazizov ${ }^{1,2, a}$, M. Kowalski ${ }^{1}$, K.S. Kuzmin ${ }^{3,4}$, V.A. Naumov ${ }^{3}$, and Ch. Spiering ${ }^{1}$ \\ ${ }^{1}$ DESY-Zeuthen, Platanenallee 6, 15738 Zeuthen, Germany \\ ${ }^{2}$ Gran Sasso Science Institute, viale Francesco Crispi, 7, 67100 L'Aquila, Italy \\ ${ }^{3}$ Joint Institute for Nuclear Research, RU-141980, Dubna, Moscow region, Russia \\ ${ }^{4}$ Institute for Theoretical and Experimental Physics, RU-117218, Moscow, Russia
}

\begin{abstract}
An updated set of (anti)neutrino-nucleon charged and neutral current cross sections at $3 \mathrm{GeV} \lesssim E_{v} \lesssim 100 \mathrm{GeV}$ is presented. These cross sections are of particular interest for the detector optimization and data processing and interpretation in the future Megaton-scale experiments like PINGU, ORCA, and Hyper-Kamiokande. Finite masses of charged leptons and target mass corrections in exclusive and deep inelastic $(\bar{v}) v N$ interactions are taken into account. A new set of QCD NNLO parton density functions, ABMP15, is used for calculation of the DIS cross sections. The sensitivity of the cross sections to phenomenological parameters and to extrapolations of the nucleon structure functions to small $x$ and $Q^{2}$ is studied. An agreement within the uncertainties of our calculations with experimental data is demonstrated.
\end{abstract}

\section{Introduction}

Future Megaton-scale neutrino detectors, such as PINGU, ORCA, and Hyper-Kamiokande can be used for determination of the neutrino mass hierarchy $(\mathrm{NMH})$ with atmospheric neutrinos. Depending on the $\mathrm{NMH}, v$-oscillations produce a few percent differences in the event rate, appearing in the few-GeV energy region, since the matter effects act oppositely for $\nu^{\prime}$ s and $\bar{\nu}^{\prime}$ s. The NMH signature relies on the fact that both fluxes and cross sections are different for $v^{\prime}$ 's and $\bar{v}$ 's, so that the combined event rates show the remaining NMH dependence. For the proper interpretation of the data, the accurate predictions of the atmospheric neutrino fluxes have to be paired with the reliable knowledge of the (anti)neutrinonucleon cross sections. We focus on the latter with an emphasis on the energy range $E_{v} \gtrsim 3 \mathrm{GeV}$ and present a self-consistent set of theoretical models and phenomenological parameters allowing us to describe the major contributions to the total $v N$ and $\bar{v} N$ cross sections at these energies. The results of the present study may be included in future upgrade of the MC generator ANIS [1] in order to extend its validity to lower energies and improve the predictions for high and ultra-high energies.

\footnotetext{
ae-mail: askhat.gazizov@desy.de
}

This is an Open Access article distributed under the terms of the Creative Commons Attribution License 4.0, which permits unrestricted use, distribution, and reproduction in any medium, provided the original work is properly cited. 


\section{Models and parameters}

All $v N$-interactions, both proceeding via charged current (CC) and neutral current (NC),

$$
\left(\bar{v}_{\ell}\right) v_{\ell}+N \rightarrow\left(\ell^{+}\right) \ell^{-}+X \quad(\mathrm{CC}) \text { and } \quad\left(\bar{v}_{\ell}\right) v_{\ell}+N \rightarrow\left(\bar{v}_{\ell}\right) v_{\ell}+X \quad(\mathrm{NC})
$$

$(\ell=e, \mu, \tau ; N=p, n)$ may be classified according to the number of mesons, pions, kaons, etc., appearing in the final hadron state $X$. The total cross sections are combinations of the contributions from the channels $(i)$ with no pions, - the (quasi)elastic scattering (ES or QES), (ii) with one pion, - the resonance single pion production (RES), and (iii) inclusive or deep inelastic scattering (DIS) with $>2$ hadrons in the final state $X: \sigma_{v N}^{\text {tot }}=\sigma_{v N}^{(\mathrm{Q}) \mathrm{ES}} \oplus \sigma_{v N}^{1 \pi} \oplus \sigma_{v N}^{\text {DIS }}$ (see, e.g., Ref. [2]).

For calculations of the (Q)ES contributions we use the standard approach with the nucleon axial mass parameter $M_{A}^{\mathrm{QES}}=1.02 \mathrm{GeV}$ extracted from available $\nu_{\mu} \mathrm{D}, \bar{v}_{\mu} \mathrm{H}$, and $\pi^{ \pm}$electroproduction data (for details, see Refs. [3-5] and references therein).

For obtaining of RES contributions the extended Rein-Seghal model [6-8] is adopted with account for the pion-pole contribution to the hadronic axial current [9]. The value of the axial mass parameter $M_{A}^{\mathrm{RES}}=1.12 \mathrm{GeV}$ has been derived in Ref. [10] by fitting to the data available at that time. All known nucleon resonances with masses below $\approx 2 \mathrm{GeV}$ are included and the interference of their amplitudes is properly taken into account according to Ref. [11].

For the DIS cross sections we use the approach of Ref. [12] with all 5 structure functions (SFs) being taken into account. In the case of CC processes this allows to account for the finite lepton mass, especially important for $v_{\tau}$. The functions $F_{1,2,3}\left(x, Q^{2}\right)$ are available by the OPENQCDRAD-2.0 code [13]; we use it with the new NNLO (both for light and charm production) parton distribution functions of ABMP15 [14]. Transitions from $F_{1,2,3}\left(x, Q^{2}\right)$ to target mass corrected (TMC) SFs, $F_{i}^{\mathrm{TMC}}\left(x, Q^{2}\right)$, are performed according to Ref. $[15,16]$. Below we do not discuss the complicated problem of the nuclear effects in the DIS SFs and focus only on purely (anti)neutrino-nucleon reactions.

To avoid a double counting, the phase spaces of RES and DIS are to be separated according to the mass of the final hadron state in Eq. (1), $W_{\text {cut }}=m_{X} \gtrsim m_{N}+2 m_{\pi}$. We found that $W_{\text {cut }}=1.4 \mathrm{GeV}$ provides a good compatibility of these contributions: a variation of $W_{\text {cut }}$ around $1.4 \mathrm{GeV}$ leads to comparatively small variations of the sum $\sigma^{\mathrm{RES}}(E)+\sigma^{\mathrm{DIS}}(E)$ at all energies.

At intermediate energies significant uncertainties in calculations of the DIS cross sections arise from necessary extrapolations of $F_{i}^{\mathrm{TMC}}\left(x, Q^{2}\right)$ to small $Q^{2}$ where the perturbative QCD fails. In accordance with the suggestion of Ref. [17], we smoothly switch all SFs off for a given $x$ as $Q^{2} \rightarrow 0$, assuming a power-law dependence on $Q^{2}$ (or on $\log \left(Q^{2}\right)$ in the case of $F_{3}^{\mathrm{TMC}}\left(x, Q^{2}\right)$ ); $F_{i}^{\mathrm{TMC}}\left(x, Q^{2}\right)=F_{i}^{\mathrm{TMC}}\left(x, Q_{\min }^{2}\right) \times\left[Q^{2} /\left(Q^{2}+b\right)\right]^{\alpha(1-x)}$. The parameter values $Q_{\min }^{2}=1.2 \mathrm{GeV}^{2}, \alpha=$ 0.5 and $b=0.645 \mathrm{GeV}^{2}$ provide a reasonable agreement with the data.

Cross section for $v_{\mu}$ and $\bar{v}_{\mu}$ scatterings off an isoscalar nucleon calculated using our set of parameters are shown in Fig. 1. Analogous plots for the $v_{\tau} N$ and $\bar{\nu}_{\tau} N$ cross sections are depicted in Fig. 2. All kinematic effects are taken into account.

In Figs. 1 and 2 the uncertainties for the QES and RES contributions are estimated by varying the QES and RES axial mass parameters within the ranges $0.9 \mathrm{GeV} \leq M_{A}^{\mathrm{QES}} \leq 1.1 \mathrm{GeV}$ and $1.1 \mathrm{GeV} \leq$ $M_{A}^{\mathrm{RES}} \leq 1.3 \mathrm{GeV}$, respectively. The upper bounds of the bands in Fig. 1 correspond to the higher values of the axial masses. In order to estimate the DIS uncertainties, we vary the parameter $\alpha$ within the range 0.3 to 0.7 .

The solid curves in Fig. 1 show similar predictions of the Monte Carlo generator GENIE [18]. The reasons of our discrepancies with the GENIE predictions at low energies are mainly due to details of the implementation of the Rein-Sehgal model [11] for the resonance single-pion production and different cuts in $W$ used in the calculations. This point will be discussed at length in a forthcoming paper. Here we only mention the effect of finite lepton mass to the leptonic currents [6], including the pion-pole contribution into the weak hadronic current [9], the effect of interference between the 

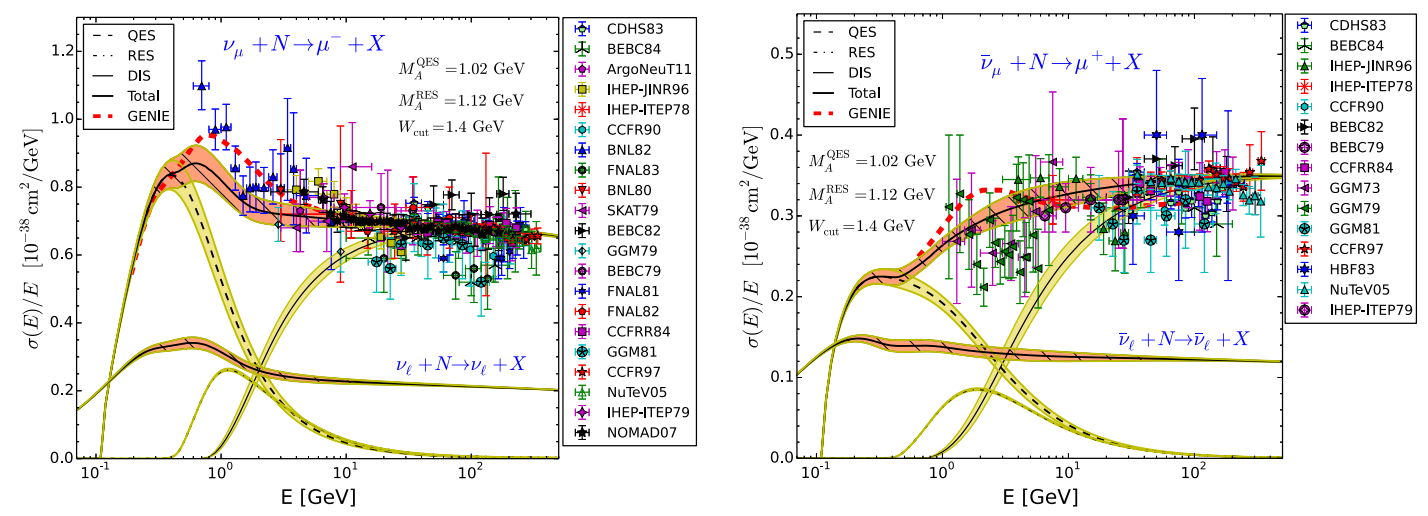

Figure 1. Left panel: Cross sections over energy for the CC $v_{\mu} N$ and NC scatterings off an isoscalar nucleon. Dashed, dash-dotted and thin solid lines correspond to contributions from QES, RES and DIS, respectively. Shaded bands show the uncertainties. Thick solid curve stands for the total cross section; the shaded hatched regions demonstrates the overall uncertainty. The thick dashed curve is the prediction of the GENIE neutrino MC generator [18]. Right panel: The same curves as in the left panel but for CC $\bar{v}_{\mu} N$ and NC scattering off the same target. The references to the data points can be found in Ref. [2].
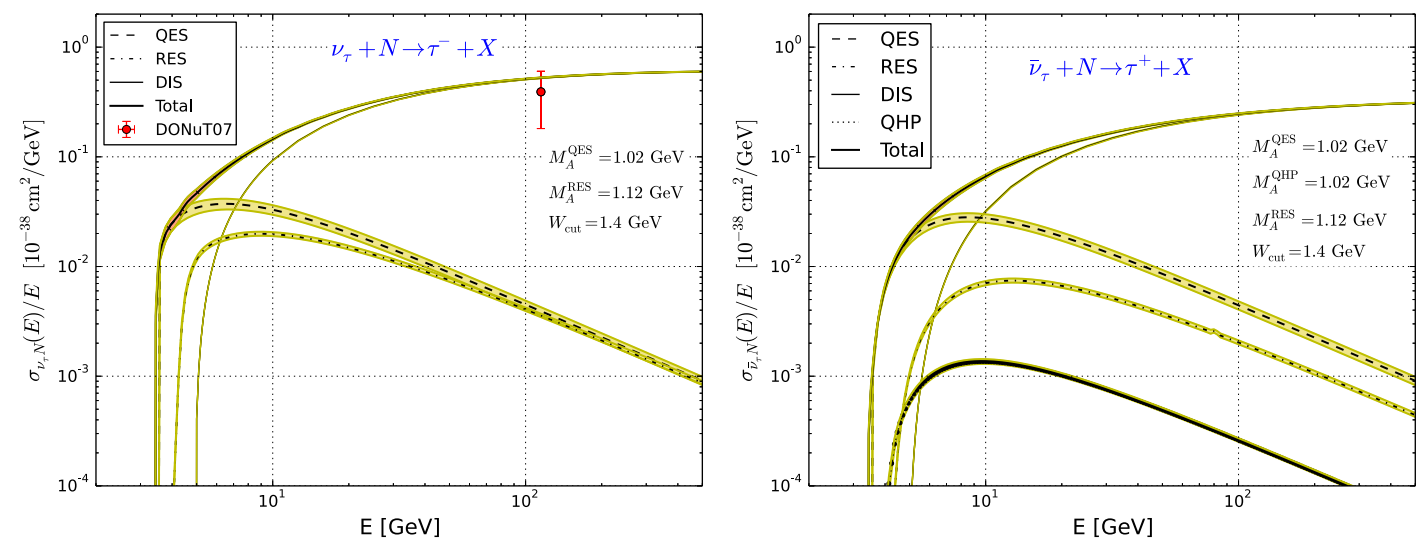

Figure 2. The same curves as in the left panel of Fig. 1 but for $\mathrm{CC} v_{\tau} N$ and $\bar{v}_{\tau} N$ scatterings. Additional dotted QHP line in the right panel shows a contribution from hypercharge violating QES production of lightest hyperons [19]. The DONUT data point is from Ref. [20].

resonances having the same spin and orbital angular momentum of the final $N \pi$ state (both these effects are neglected in the cited version of GENIE), and also different "recipes" used for normalization of the Breit-Wigner (BW) factors and treatment of the unphysical BW "tails". The disagreements with GENIE at high energies (most notable for $\bar{v}$ ) are mainly due to the PDF models involved in the GENIE code and in our calculations, and due to distinct methods for extrapolating the DIS SFs to small $Q^{2}$.

\section{Conclusions}

We presented a comprehensive set of the total cross sections for the neutrino and antineutrino scattering off protons and neutrons at energies most important for the future experiments with Mton-scale neutrino detectors. Contributions from elastic and quasi-elastic scatterings (with the hypercharge change $\Delta Y=0$ and 1), single-pion production, and deep-inelastic scattering were taken into account. For 
the ES, QES, and single-pion production contributions we rely on well-known methods and models. With the reasonable choice of parameters, our calculations are in reasonable agreement with the data within the experimental uncertainties. Calculations of the DIS cross sections were performed by using OPENQCDRAD-2.0 with the new set of NNLO PDFs of ABMP15 with account for the finite lepton masses and target mass corrections. We find that the uncertainties due the choice of the SFs extrapolations to small $Q^{2}$ are very significant. This partially explains the disagreements between our model and the calculations using the GENIE packet at high energies.

Present calculations will be used as a basis for the future upgrade of the Monte Carlo neutrino generator ANIS [1].

We are grateful to S. Alekhin, J. Blümlein and S.-O. Moch for making us available the new OPENQCDRAD and ABMP15 PDFs and to I. Kakorin for a valuable contribution. A.G. thanks the Helmholtz Alliance for Astroparticle Physics, HAP for support. The work of K.K. and V.N. was partially supported by the Russian Foundation for Basic Research under Grant No. 14-22-03090.

\section{References}

[1] A. Gazizov, M.P. Kowalski, Comput. Phys. Commun. 172, 203 (2005), astro-ph/0406439

[2] K.S. Kuzmin, V.V. Lyubushkin, V.A. Naumov, Phys. Atom. Nucl. 69, 1857 (2006)

[3] V. Bernard, L. Elouadrhiri, U.G. Meißner, J. Phys. G28, R1 (2002), hep-ph/0107088

[4] A. Bodek, S. Avvakumov, R. Bradford, H.S. Budd, Eur. Phys. J. C53, 349 (2008), 0708.1946

[5] K.S. Kuzmin, V.V. Lyubushkin, V.A. Naumov, Eur. Phys. J. C54, 517 (2008), 0712.4384

[6] K.S. Kuzmin, V.V. Lyubushkin, V.A. Naumov, Mod. Phys. Lett. A19, 2815 (2004)

[7] K.S. Kuzmin, V.V. Lyubushkin, V.A. Naumov, Nucl. Phys. B (Proc. Suppl.) 139, 158 (2005)

[8] D. Rein, L. Sehgal, Phys. Lett. B657, 207 (2007), hep-ph/0606185

[9] C. Berger, L. Sehgal, Phys. Rev. D76, 113004 (2007); Erratum: ibid., D77, 059901(E) (2008)

[10] K.S. Kuzmin, V.V. Lyubushkin, V.A. Naumov, Acta Phys. Polon. B37, 2337 (2006)

[11] D. Rein, L.M. Sehgal, Annals Phys. 133, 79 (1981)

[12] C.H. Albright, C. Jarlskog, Nucl. Phys. B84, 467 (1975)

[13] https://www-zeuthen.desy.de/ alekhin/OPENQCDRAD/ (2012)

[14] S. Alekhin, J. Blümlein, S. Moch, R. Plačakyte (2015), arXiv:1508.07923 [hep-ph]

[15] S. Kretzer, M. Reno, Phys. Rev. D66, 113007 (2002), hep-ph/0208187

[16] S. Kretzer, M. Reno, Phys. Rev. D69, 034002 (2004), hep-ph/0307023

[17] A. Capella, A. Kaidalov, C. Merino, J. Tran Thanh Van, Phys. Lett. B337, 358 (1994)

[18] C. Andreopoulos et al., Nucl. Instrum. Meth. A614, 87 (2010), 0905.2517

[19] K.S. Kuzmin, V.A. Naumov, Phys. Atom. Nucl. 72, 1501 (2009)

[20] E.O. Maher (FERMILAB-THESIS-2005-90, UMI-31-92032), 2008 